\title{
Measurement and evaluation of hand-transmitted vibrations by means of their powers
}

\author{
Toshisuke Miwa \\ National Institute of Industrial Health, \\ 21, 1 Nagao 6-chome, Tama-ku, Kawasaki, 213 Japan \\ (Received 19 January 1988)
}

\begin{abstract}
A vibration power pickup and its power-calculation circuits were made for use in field surveys and a method of calibrating the instrument was devised. In order to develop methods of evaluating the vibration power generated from hand-held vibrating power tools, psychological equal-sensation experiments were carried out, using continuous sinusoidal motions in the frequency range of $8-500 \mathrm{~Hz}$ and bursts with various cycles of a $31.5-\mathrm{Hz}$ fixed frequency. Rating experiment were also done involving five successive categories of power level (PWL) ranging from 100 to $125 \mathrm{dBPWL}$. The vibration power levels of a rock-drill, a breaker and an electric grinder were measured using the power meter and their effects on human operator were estimated.
\end{abstract}

PACS number: 43. 40. $\mathrm{Ng}$

\section{INTRODUCTION}

Vibration-induced health hazard problems among workers using vibrating hand-held power tools such as chain saws, rock-drills, chipping hammers and grinders have attracted considerable attention. An instrument for vibration measurement has been standardized in Japan as JIS C 1511, "Vibration level meter for portable vibrating tools" together with the measuring method, JIS B 4900, "Measuring method for the tool vibration levels of portable power tools." The tool vibration level has been defined in Japan as the frequency-weighted vibration acceleration level of power tools. The frequency characteristics proposed in ISO 5349 have been used as the frequency weighting. Consequently, vibration measurement has been facilitated and comparisons of results obtained by different researchers have become possible. However, the level of vibration acceleration measured on the handles of power tools by the methods described in these standards is not adequate for the evaluation of hand-transmitted vibration because the handle acceleration is not fully transmitted to the human hand due to the hand gripping force, working posture and the mechanical impedance of the hand. We have found that the vibration force transmitted to the human hand can be treated as a distinct vibration quantity, which genuinely reflects the working and mechanical conditions of the hand. If the vibration power value can be derived from the vibration velocity integrated from the acceleration and vibration force values, this single value will hopefully be an accurate representation of the true vibration quantity entering the worker's hands.

Several papers have been published on vibration power measurement. Lidstroem ${ }^{1)}$ in her pioneering work made a special handle containing a force pickup and an accelerometer which was placed in contact with the handles of vibrating tools in order to measure the power of vibration. She observed a relationship between the vibration power and vibration-induced white finger (VHF).

Reynolds et al. ${ }^{2)}$ measured vibration energy by an indirect method. For this, the driving-point dynamic compliance of the hand and the vibration acceleration spectra at the tool handle were measured in three directions respectively and the vibration 
energy per unit time was calculated. They demonstrated the dissipative energy absorbed in the part of the hand showing resistance, the stored energy in kinetic and potential components in the spring and mass elements in the hand, and the total energy.

We noticed the effective vibration power $(F V \cos \varphi)$ (dissipative component) in one vibrational direction for the purpose of field surveys in working circumstances, so that any measuring system would have to be simple and portable. In the present study, a prototype vibration power meter was produced using an analogue circuit and a method for calibrating the meter was developed. Using this vibration power meter, psychological experiments were carried out in order to obtain a vibration evaluation method. Finally, using the method described, the vibration power was measured and evaluated for a rock-drill with a leg, a breaker and an electric grinder as an example of the situations encountered in field surveys.

\section{EXPERIMENTS}

2.1 Production, Calibration and Response of Power Meter

\subsubsection{Vibration power meter}

A vibration power pickup $50 \times 25 \times 9 \mathrm{~mm}$ in size was devised, comprising two force transducers (16 $\mathrm{mm}$ in diameter and $5 \mathrm{~mm}$ thick, Rion Co.) sandwiched between two bakelite plates ( $2 \mathrm{~mm}$ thick) and an acceleration transducer $(16 \mathrm{~mm}$ in diameter and $4 \mathrm{~mm}$ thick, Rion Co.) placed in the center. Each element was set in epoxy resin adhesive and
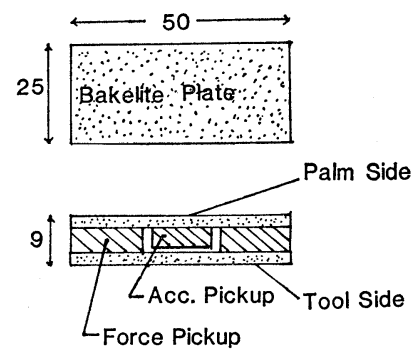

Fig. 1(a) Vibration power pickup (length unit in $\mathrm{mm}$ ). Its cross-section is $9 \times 25 \mathrm{~mm}$.

the side directly connected to the accelerometer was applied to the palm (Fig. 1(a)). The functions of the circuits in the vibration power meter are shown in the form of a block diagram (Fig. 1(b)). The signals of the vibration acceleration $(A)$ and the vibration force $(F)$ were amplified by two channel vibration level meters (VM 17 Rion) and the acceleration signal was converted into the velocity $(V)$ by an integrated circuit. One of the force and velocity signals after amplification was rectified to an r.m.s. value and converted into a logarithmic quantity, both calculations being carried out by one AD536A integrated circuit (IC), and indicated by an ammeter $(500 \mu \mathrm{A})$. The indicator showed the r.m.s. level (dB) of acceleration $L_{\mathrm{A}}=20 \log _{10} A_{\mathrm{rms}} / 10^{-5} \quad$ (vibration acceleration level dBAL), that of velocity $L_{\mathrm{V}}$ $=20 \log _{10} V_{\mathrm{rms}} / 10^{-9}$ and that of force $L_{\mathrm{F}}=$ $20 \log _{10} F_{\mathrm{rms}} / 10^{-6}{ }^{3)}$ where $A_{\mathrm{rms}}$; rms vibration accleration value $\mathrm{m} / \mathrm{s}^{2}, V_{\mathrm{rms}} ;$ rms vibration velocity value $\mathrm{m} / \mathrm{s}$ and $F_{\mathrm{rms}} ;$ rms vibration force value $N$.

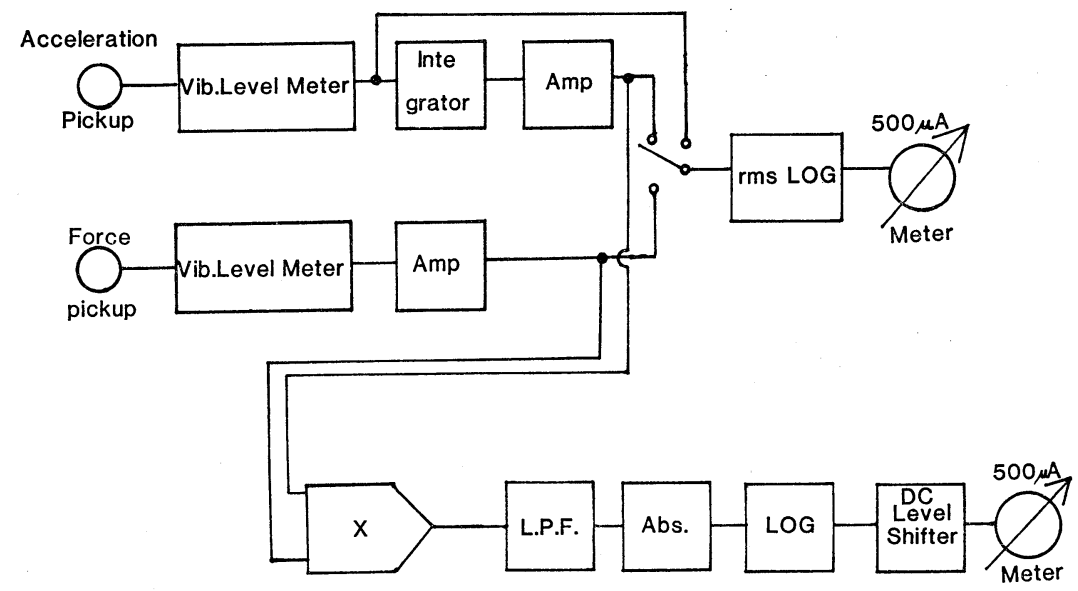

Fig. 1(b) Block diagram of vibration power meter. 


\section{T. MIWA: POWER MEASUREMENT AND EVALUATION OF HAND VIBRATIONS}

The value of $10^{-5}$ is adopted as $A_{\text {ref }}$ in dB because this value has been adopted in Japan instead of $10^{-6}$ in ISO. The force and velocity signals were fed into a multiplier (AD534J) for calculation of $F V \cos \varphi$ as a D.C. component, which was filtered out by a low-pass filter (L. P. F.) constructed from an integrator circuit. The time constant of the integrator determined the speed of the indicator. The power was also converted into a logarithmic value by a $759 \mathrm{~N}$ IC as $10 \log _{10} W / 10^{-12}(\mathrm{dBPWL})$ via an absolute value calculator and a D. C. level shifter and indicated by another ammeter $(500 \mu \mathrm{A})$.

Consequently, the power level described above is defined as the vibration power passing through the cross-section of power pickup $(50 \times 25 \mathrm{~mm})$.

\subsubsection{Calibration of vibration power meter}

The acceleration, velocity and force were individually calibrated on the electro-dynamic vibrator using the known acceleration level, the weight and the vibration frequency. The power pickup was set on the vibrator, on which a $0.5-\mathrm{kg}$ weight was placed. A $50-\mathrm{Hz}$ r.m.s. $10 \mathrm{~m} / \mathrm{s}^{2}(120-\mathrm{dB})$ vibration acceleration was generated on the vibrator, so that a vibration force of $0.5 \mathrm{~kg} \times 10 \mathrm{~m} / \mathrm{s}^{2}=$ r.m.s. $5 \mathrm{~N}$ $(134 \mathrm{~dB})$ was observed and a vibration velocity of r.m.s. $10 /(2 \pi 50) \mathrm{m} / \mathrm{s} \quad(150 \mathrm{~dB})$. The meter was calibrated using these values. A mechanical system with a single degree of freedom was constructed with a mass $(0.5 \mathrm{~kg})$ and a visco-elastic material (vinyl chloride foam, $50 \mathrm{~mm}$ in diameter, $30 \mathrm{~mm}$ thickness), which acted as a spring and provided mechanical resistance. The power pickup was set on the vibrator, on which this resonance system was placed with an interposing aluminum disk $(50 \mathrm{~mm}$ in diameter and $2 \mathrm{~mm}$ thickness) which was negligible for the power value of this system. The phase between the velocity and the force signals was monitored by synchroscope in order to detect the resonance ( 0 phase). The vibration power given to the system can be calculated at the resonance point as:

$$
W=\left(V_{\mathrm{rms}}\right)\left(F_{\mathrm{rms}}\right) .
$$

The vibration power level $(P W L)$ is determined using Eq. (1) as:

$$
\begin{aligned}
P W L & =10 \log _{10}\left(F_{\mathrm{rms}} V_{\mathrm{rms}} / 10^{-12}\right) \\
& =(1 / 2)\left(L_{\mathrm{V}}+L_{\mathrm{F}}\right)-30 .
\end{aligned}
$$

\subsubsection{Response of random vibration}

The vibration power of sinusoidal motion can be measured, but it is necessary to check the response of the power meter to random motion for application in a field survey. An M-sequence pseudo random motion was generated on the vibrator using a frequency equalizer which corrected the frequency characteristics of the vibrator so that they were flat. The power pickup was placed on the vibrator, on which the resonance system mentioned above was placed. The cross correlogram between the force and velocity signals was calculated with a signal analyzer (SM-2100-A Iwatsu Co.). The vibration power level in the resonance system was then compared with the cross-correlogram at $t=0(t=$ delay time).

\subsection{Psychological Experiments}

Ten male subjects ranging in age from 25 to 50 years participated in the psychological experiments. An aluminum cylindrical bar $(30 \mathrm{~mm}$ in diameter and $200 \mathrm{~mm}$ long) was set on the vibrator using two square pillars $(30 \times 30 \times 60 \mathrm{~mm})$ as a gripping handle simulating that of a power tool. The power pickup was set on the handle using dental impression material (GC Ostron 100, GC Dental Industry Co.). The subject was asked to grip the handle with the right hand. The static forces involved in pushing and gripping were measured using foam rubber with electrical conductance which changed in proportion to the pressing force. The static force pickup was made from strips of both this rubber and ordinary foam rubber, in order to strengthen the spring force, set alternately and sandwiched between two phosphor-bronze plates $(45 \times 18 \times 0.3 \mathrm{~mm}$ ) (Fig. 1(c)). Two electric wires were soldered onto the plates separately, and were connected to the conduction tester (Ohm meter). The total thickness of the pickup was $1.5 \mathrm{~mm}$. The static force was calibrated using a spring balance upon application of known weights. One of the static force pickups was placed on the power pickup and the other on

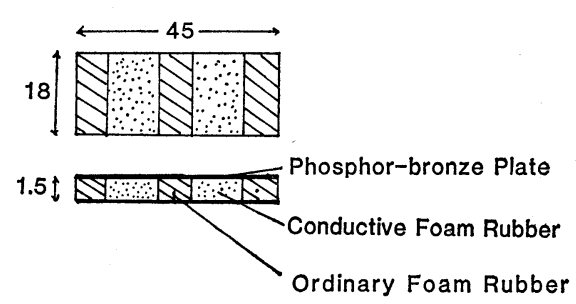

Fig. 1(c) Static force pickup. Its crosssection is $1.5 \times 18 \mathrm{~mm}$. 
the other side of the handle. The former was considered to show the sum of both the pushing and gripping forces and the latter the gripping force alone. The pushing force was derived by an electric circuit subtracting the gripping force from summation of both forces. As the power level varied easily according to the pushing and gripping forces applied to the handle, the subject was required to maintain the pushing force at nearly $20 \mathrm{~N}$ and the gripping force at nearly $35 \mathrm{~N}$ by watching the two ohm meters during the experiments. The tester also monitored the power meter for any fluctuation and sometimes asked the subject to check his pushing and gripping forces and/or posture in order to maintain a constant power level appointed.

\subsubsection{Equal sensation to sinusoidal motions}

The subject was asked to compare two vibrations, the reference and the test, given sequentially in order to obtain the equal sensation. A $31.5-\mathrm{Hz}, 105-$ dBPWL sinusoidal vibration corresponding to about $110 \mathrm{dBAL}$ was chosen as the reference stimulus, and sinusoidal vibrations at six octave center frequencies between 8 and $500 \mathrm{~Hz}$ were examined as the test stimuli. One sequence consisted of a 5 -s reference motion, a 2-s pause and a 5-s test motion. After a further 2-s pause, the same sequence was repeated, but the level of the test vibration was varied. This sequence was continued until the subject was able to feel a sensation equal to that of the reference. Then, the vibration frequency was changed.

The level of the test motion was varied by the tester (method of adjustment by the tester) using a $60-\mathrm{dB}$ attenuator with $1-\mathrm{dB}$ steps according to the response of the subject, i.e. "larger" or "smaller." For an ascending series, the increase in the level was rough until it neared the predicted equal sensation point, after which care was taken to determine the exact point of equal sensation. The descending series was carried out using the same procedure as the ascending series and their results were averaged. The level of equal sensation judged was measured by the power meter. The frequency and the initial level of the test motion applied to the subject were changed randomly.

\subsubsection{Equal sensation to bursts}

This experiment was carried out to collect information on the averaging time of the integrating circuit (L. P. F.) of the vibration power meter. A burst was generated by a burst-signal generator, the fundamental frequency of which was kept constant at
$31.5 \mathrm{~Hz}$ but the duration of the burst was changed from 2 to 63 cycles of a $31.5-\mathrm{Hz}$ sinusoidal signal. The level of the bursts was always maintained constant at $123 \mathrm{dBAL}$ peak (peak vibration acceleration level) at each of seven durations, i.e. 2, 3, 4, 8, 16,31 and 63 cycles of $31.5 \mathrm{~Hz}$. A sequence consisting of the burst motion, a 2-s pause and a 5-s, $5-\mathrm{Hz}$ continuous sinusoidal motion was applied to the subject. The subject was required to compare the feeling of a burst with that of continuous motion. Following the subject's response of "larger" or "smaller," the level of continuous vibration was adjusted by the tester in order to obtain the sensation equal to a burst of motion. This sequence was repeated until the subject reached an equal sensation. An ascending and a descending series were performed using the same procedure mentioned previously and their results were averaged. The durations of the bursts and the initial level of continuous motion in the ascending and descending series were changed randomly. The peak acceleration level of a burst was measured by the peak hold meter (VM-20-A Rion Co.) and an equal sensation level of $31.5 \mathrm{~Hz}$ continuous motion judged was observed using the power meter.

\subsubsection{Rating of five successive categories}

To determine the relation between the feeling of vibrations in the hand and their power levels, a rating experiment was carried out. The tester asked the subject to imagine being under working conditions where he was using a power tool. A 5-s, 31.5$\mathrm{Hz}$ continuous sinusoidal vibration with a certain level was applied twice to the subject separated by a 2-s pause. The subject was required to assess the unpleasantness of the feeling of vibration on a scale assigned five successive categories. The frequency was kept constant at $31.5 \mathrm{~Hz}$, while the power level of the vibration tested was randomly changed from 100 to $125 \mathrm{~dB}$ at $5-\mathrm{dB}$ intervals. Using a scale of $1,2,3,4$ and 5 for each category, the rating scale was defined as 1 (not unpleasant), 2 (slightly unpleasant), 3 (mildly unpleasant), 4 (moderately unpleasant) and 5 (severely unpleasant). The experiments were repeated twice and their results were averaged.

\section{RESULTS AND DISCUSSION}

\subsection{Response of Random Vibration}

A cross-correlogram for the single resonance system exposed to random vibration is shown in Fig. 2. Generally speaking, the cross-correlogram 


\section{T. MIWA: POWER MEASUREMENT AND EVALUATION OF HAND VIBRATIONS}

of this resonance system indicates a peak or notch at $t=0$. The power level read from the power meter agreed well with that obtained from the correlogram at $t=0$ (vertical line in the figure), that is, $15 \mathrm{~mW}=102 \mathrm{dBPWL}$. It was thus clarified that the power meter was able to respond correctly even for random vibration.

\subsection{Equal Sensation to Sinusoidal Motions}

Figure 3 shows the results of equal sensation for sinusoidal motion at the center frequencies of the octave band from 8 to $500 \mathrm{~Hz}$. The abscissa is the frequency $(f)$ and the ordinate is the vibration power level $(P W L)$ observed. A $31.5-\mathrm{Hz}, 105-\mathrm{dB}$ PWL sinusoidal motion was taken as the reference, and the equal sensations in power level observed are shown as different symbols for the 10 participants. It can be seen that the divergence of the observed values did not exceed $\pm 6 \mathrm{~dB}$. The quadratic equation (representing the solid line) was calculated by the least squares method as:

$$
y=119.4-19.7(\log f)+6.9(\log f)^{2} .
$$

For convenience in making the frequency weighting

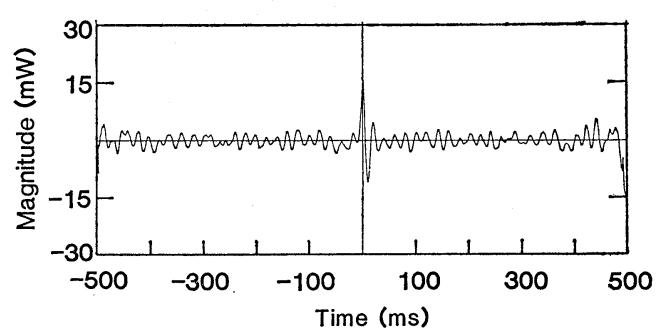

Fig. 2 Cross-correlogram of the resonance system.

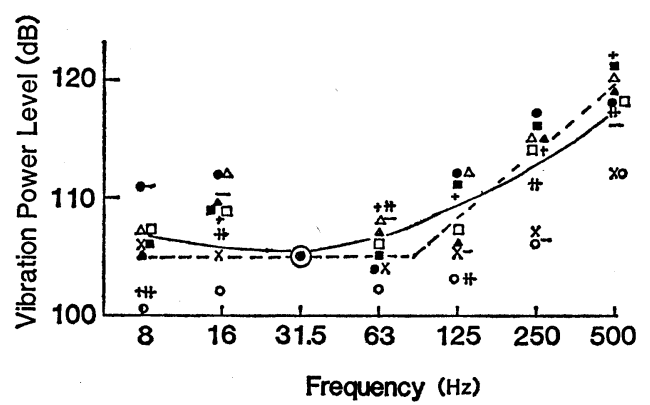

Fig. 3 Equal sensation curve for sinusoidal vibration. circuit, another approximate curve was considered (broken line), the frequency characteristics of which were similar to a low-pass filter with a $125-\mathrm{Hz}$ cutoff frequency ( $-3 \mathrm{~dB}$ down).

Reynolds et al. concluded that the power values above $20 \mathrm{~Hz}$ decrease with increasing frequency. This fact can be explained in terms of vibration transmission and vibration perception problems. The transmission of vibration force to the human hand decreases with increasing frequency and the velocity also decreases with increasing frequency by $1 /(2 \pi f)$ with regard to the vibration acceleration. The vibration receptors of the human hand have decreased sensitivity for higher frequencies due to decreasing vibration amplitude. In our previous experiment concerning equal sensation felt by the hand, the curves with vibration acceleration as the ordinate showed a slope of $12 \mathrm{~dB} /$ oct. above $80 \mathrm{~Hz}{ }^{4}$ ) This finding hints the equal sensation curve with regard to power level, the slope of which is $6 \mathrm{dBPWL} /$ oct.

\subsection{Equal Sensation of Bursts}

Figure 4 shows the equal sensation felt in response to bursts composing 7 different cycles of $31.5 \mathrm{~Hz}$. The ordinate indicates the attenuation $\left(y=y^{\prime}-120\right.$, dB) where $y^{\prime}$ dBPWL represents the vibation power level judged as the equal sensation, and for convenience of calculation, 120 is subtracted from the values of $y^{\prime}$ PWL. The abscissa indicates $x=$ $\log _{10} N$ where $N$ is the number of cycles of $31.5-\mathrm{Hz}$ sinusoidal vibration, so that the abscissa does not indicate the real duration time $(N / 31.5)$ for convenience of calculation of the regression. It can be observed that the curve did not reach 0 attenuation even at 62 cycles $(2 \mathrm{~s})$, remaining below it by about

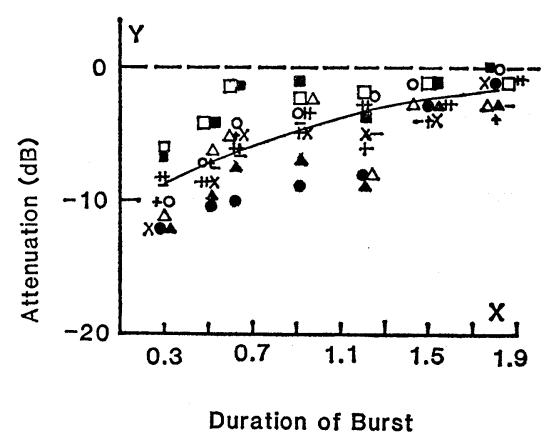

Fig. 4 Equal sensation curve for bursts. 
$2 \mathrm{~dB}$. The regression curve was determined by the least squares method as:

$$
y=-11.27+9.1 x-2.24 x^{2} .
$$

From a practical viewpoint, the time constant of the integrator in L. P. F. of the indicator component was determined as $2 \mathrm{~s}$.

\subsection{Rating of Five Successive Categories}

Figure 5 shows the results of the rating experiment on 5 successive categories. The ordinate is the number of categories and the abscissa represents the value of $x=x^{\prime}-100(\mathrm{~dB})$, where $x^{\prime} \mathrm{dBPWL}$ is the vibration power level applied to the subject and 100 is subtracted from the values of $x^{\prime}$ for convenience of calculation. The divergence of the observed data is similar at each power level. This result means that the subjects did not feel these vibrations to be quite severe even at the maximum level of 125 dB. When 125-dB motion was felt quite severely, the observed values tended to be clustered at one point in category 5 . The linear regression was calculated by the least squares method as:

$$
y=0.78+0.14 x,
$$

where $y$ is the category number.

\subsection{Field Survey}

The vibration power level was measured for a leg-type rock-drill driven by air pressure of $5 \mathrm{kgf} /$ $\mathrm{cm}^{2}$, a breaker driven by air pressure of $6 \mathrm{kgf} / \mathrm{cm}^{2}$ and a grinder powered by electricity at $\mathrm{AC} 100 \mathrm{~V}$. The power pickup was set in the center of the palm of the right hand of each worker using sticking plaster. The breaker was placed on a concrete block for vertical boring. The rock-drill was

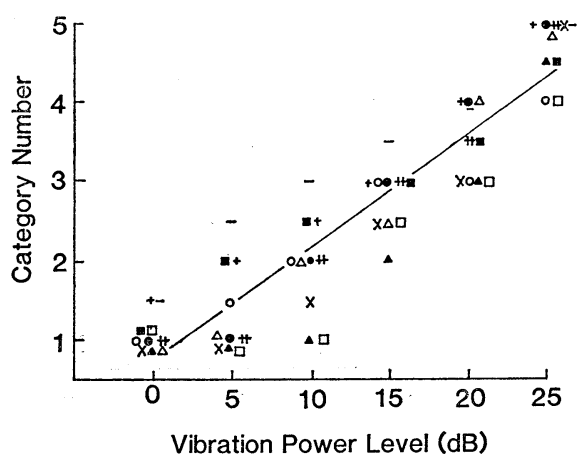

Fig. 5 Rating of five successive categories.
Table 1 Working conditions for hand-held power tools and their maximum vibration power levels observed after reaching

\begin{tabular}{|c|c|c|c|}
\hline & Rock-drill & Breaker & Grinder \\
\hline Isolator & With & Without & Without \\
\hline Blowing N. & 1,800 b.p.m. & 1,100 b.p.m. & \\
\hline Round N. & & & $50 \mathrm{~Hz}$ \\
\hline Object & $\begin{array}{l}\text { Granite } \\
\text { block } \\
1,000 \times 1,020 \\
\times 1,500 \mathrm{~mm}\end{array}$ & $\begin{array}{l}\text { Concrete } \\
\text { block } \\
1,070 \times 1,050 \\
\times 1,400 \mathrm{~mm}\end{array}$ & $\begin{array}{l}\text { Iron disc } \\
300 \times 30 \mathrm{~mm}\end{array}$ \\
\hline Work & $\begin{array}{l}3 \text { holes } \\
\text { boring }\end{array}$ & $\begin{array}{l}3 \text { holes } \\
\text { boring }\end{array}$ & 5 min \\
\hline Grip F. & $37 \mathrm{~N}$ & & $\begin{array}{l}50 \text { N Left } \\
30 \text { N Right }\end{array}$ \\
\hline Push F. & $23 \mathrm{~N}$ & $250 \mathrm{~N}$ & \\
\hline $\begin{array}{l}\text { Power L. } \\
\text { (Max) } \\
\text { (dBPWL) }\end{array}$ & 120 Right & 130 Right & $\begin{array}{l}106 \text { Right } \\
113 \text { Left }\end{array}$ \\
\hline
\end{tabular}
stable steady state.

placed beside a granite block for horizontal boring. The vibration power was also measured during grinding work using an iron disc at the left hand palm. The static force of gripping and pushing was measured using the same procedure as described above. The working conditions and results are tabulated in Table 1. The power level of the breaker without a vibration isolation handle was about 130 dBPWL at the right hand. That of the rock-drill with a isolation handle at the right hand was $10 \mathrm{~dB}$ less. That of the grinder was about $113 \mathrm{dBPWL}$ at the left hand and $106 \mathrm{dBPWL}$ at the right hand. These results agree approximately with those obtained by Lidstroem. For evaluation of vibrations by their power levels, although those of rock-drills and breakers involve shock-motion, the shocks were repetitive, so that they can be considered to involve continuous vibration. Since the main frequency of a rock-drill is $30 \mathrm{~Hz}$ and that of a breaker is about $18 \mathrm{~Hz}$, Fig. 5 shows that it is not necessary to correct their power levels according to the frequency weighting. In the case of a grinder, correction is also unnecessary because the main frequency is between 80 and $100 \mathrm{~Hz}$. From the results of the rating experiment shown in Fig. 5, an approximately 110-dBPWL grinder corresponds to scale 2 "slightly unpleasant," a rock-drill of about 120 dBPWL to scale 4 "moderately unpleasant" and a breaker of about $130 \mathrm{dBPWL}$, since it is over 125 


\section{T. MIWA: POWER MEASUREMENT AND EVALUATION OF HAND VIBRATIONS}

dBPWL, may belong to scale 5, "severely unpleasant."

The power level is influenced by the gripping and pushing force applied to the handle, the mechanical structure of the individual human hand, the size of the power pickup and its location on the palm, and the angle between the axis of the pickup and the vibration direction. Many carefully repeated measurements will be necessary on the same tools and a field survey will have to be carried out for many other types of hand-held power tools. On the other hand, standardization of the size of the power pickup, its location on the palm, and the magnitude of the pushing and gripping forces will be necessary in order to obtain high reproducibility and compatibility among different researchers for measuring the power levels of hand-held power tools.

\section{ACKNOWLEDGEMENT}

The author is indebted to Dr. S. Koshi of the director of this institute, for his kind advice. Thanks are also extended to the staff of the design section in the rock-drill production department of Furukawa Mining Co. for their generous assistance in carrying out measurements using the rock-drill and the breaker.

\section{REFERENCES}

1) I. M. Lidstroem, "Vibration injuries in rock drillers, chiselers and grinders," NIOSH Publication No. 77-170, 77-83 (1978).

2) D. D. Reynolds, R. Basel, D. E. Wasserman, and W. Taylor, "A study of hand vibration in chipping and grinding operators. Part III. Power level into the hands of operators of pneumatic tools used in chipping and grinding operations," $\mathbf{J}$. Sound Vib. 95, 515-524 (1984).

3) ISO 1683, "Acoustics-preferred reference quantities for acoustic levels" (1983).

4) T. Miwa, "Evaluation methods for vibration effect. Part 3. Measurement of threshold and equal sensation contours on hand for vertical and horizontal sinusoidal vibrations," Ind. Health 5, 213220 (1967). 